\title{
Parâmetros bioquímicos e hemogasométricos do sangue total canino armazenado em bolsas plásticas contendo CPDA-1 e CPD/SAG-M
}

\author{
Parameter biochemical and hemogasometric of the total blood stored of dogs in plastic stock \\ markets I contend CPDA-1 and CPD/SAG-M
}

\author{
Jair Costa Júnior ${ }^{I}$ José Antônio VianaI José Dantas Ribeiro Filho ${ }^{I}$ Evandro Silva Favarato ${ }^{\mathrm{I}}$ \\ Lukiya Campos Mata ${ }^{I}$ Napoleão Argôlo Neto ${ }^{I}$
}

\section{RESUMO}

O presente trabalho teve como objetivo avaliar $e$ comparar as alterações bioquímicas e hemogasométricas do sangue total canino armazenado em bolsas CPDA-1 e CPD/ SAG-M. Foram utilizados 14 cães machos, adultos e saudáveis, distribuídos em dois grupos com sete animais cada, dos quais foi obtido sangue para o estudo. No primeiro grupo (G1), o sangue foi armazenado em bolsas CPDA-1 e, no segundo grupo (G2), o sangue foi armazenado em bolsas CPD/SAG-M. As amostras foram analisadas para: potássio, sódio, glicose, proteína plasmática total, DPG, $\mathrm{pH}$ sangüíneo, $\mathrm{pO}_{2}, \mathrm{pCO}_{2} e$ bicarbonato. Os momentos estabelecidos para as análises laboratoriais foram: D0: imediatamente após a coleta do sangue dos animais; D7: sete dias após a coleta; D14: quatorze dias após a coleta; D21: vinte e um dias após a coleta; D31: trinta e um dias após a coleta; D41: quarenta e um dias após coleta. A análise dos resultados permitiu concluir que a bolsa CPD/SAG-M apresentou melhor desempenho quando comparada à CPDA-1 após 41 dias de estocagem. O DPG, mesmo com $\mathrm{pH}$ inferior a sete, continua sendo produzido por, pelo menos, uma semana após a estocagem do sangue na bolsa.

Palavras-chave: sangue, CPDA-1, CPD/SAG-M, cão, conservação do sangue.

\section{ABSTRACT}

The present study was aimed at evaluating and comparing the biochemical and haemogasometrics alterations of the total blood of dogs stored in bags CPDA-1 and CPD/ SAG-M. 14 male's dogs, adult and healthful dogs, were divided in two groups, which one blood was removed for the study. The blood of the first group (G1) was stored in bag CPDA-1 and that of the second group (G2) in bag CPD/SAG-M. The samples were analyzed for: potassium, sodium, glucose, total plasmatic protein, $\mathrm{DPG}, \mathrm{pH}, \mathrm{pO}_{2}, \mathrm{pCO}_{2}$, and bicarbonate. The moments for the laboratory analyses were established: D0: immediately after the collection of blood; D7: seven days after the collection; D14: fourteen days after the collection; D21: twenty and one days after the collection; D31: thirty and one days after the collection; D41: forty one days after collect. The evaluation of the results allowed including that: the bag CPD/SAG-M presented advantage when compared with the CPDA-1 after 41 days of stored. The DPG, even with $\mathrm{pH}$ lower then 7 , continues being produced for, at least, one week after the stocking of the blood in the bag.

Key words: blood, CPDA-1, CPD/SAG-M, dog, blood conservation.

\section{INTRODUÇÃO}

A transfusão sangüínea é considerada, hoje em dia, uma forma de terapia emergencial, operando como um transplante, tendo em vista que se trata de um tecido transportado para outro indivíduo (NUSBACHER, 1994; KRISTENSEN \& FELDMAN, 1997; ROZANSKI \& LAFORCADE, 2004). Comumente a transfusão sangüínea é indicada para o tratamento de anemias severas, além da reposição dos fatores de coagulação (CHARAMONTE, 2004; LUCAS et al. 2004). Segundo BROOKS (1992), a transfusão sangüínea é considerada um transplante tecidual temporário.

Embora se conheçam as técnicas de separação dos componentes sangüíneos, a terapia com sangue total ainda é uma realidade em grande parte dos centros veterinários, sendo considerada como a “viga-mestra” na terapia sangüínea nestes lugares (KRISTENSEN \& FELDMAN, 1997; LUCAS et al., 2004).

'Departamento de Veterinária, Universidade Federal de Viçosa (UFV), 36570-000, Viçosa, MG, Brasil. E-mail: jairdjr@hotmail.com. *Autor para correspondência. 
A estocagem sangüínea para transfusão é praticada em medicina humana desde que a primeira solução preservativa, o citrato ácido dextrose (ACD), foi desenvolvida em 1943. Subseqüentemente, na área médica, muitos trabalhos têm sido realizados na tentativa de desenvolver meios para manter a viabilidade e a capacidade funcional do sangue estocado por longos períodos. A estocagem em soluções anti-coagulantes objetiva manter a viabilidade e a função de cada constituinte sangüíneo. Além disso, busca prevenir alterações físicas prejudiciais nos seus componentes e evitar a proliferação bacteriana (AUTHEMENT et al., 1986). A viabilidade do sangue estocado está na dependência da técnica da coleta, do anti-coagulante, da temperatura de conservação, dos parâmetros bioquímicos e, inclusive, da freqüência de homogeneização durante o armazenamento (WILLER \& RIEDESEL, 1985; AUTHEMENT et al., 1986; LOPES et al., 1995; HÖGMAN et al., 2002).

O ácido 2,3-difosfoglicerato (DPG) é uma molécula presente no interior dos eritrócitos e que se liga à subunidade $\beta$ da hemoglobina. O DPG é responsável pela liberação do oxigênio $\left(\mathrm{O}_{2}\right)$ para os tecidos (BUNN, 1971; EISENBRANDT \& SMITH, 1973; WARDROP et al., 1994; SCOTT et al., 2005). Desse modo, quanto menor os níveis do DPG, maior será a afinidade da hemoglobina pelo $\mathrm{O}_{2}$. Conseqüentemente, mais difícil será a liberação deste para os tecidos.

$\mathrm{O}$ principal fator relacionado à redução do DPG é o pH. Níveis de $\mathrm{pH}$ maiores de que sete são tidos como propícios para a regeneração do DPG. Valores inferiores a sete, favorecem a degradação do DPG, contribuindo de forma significativa na deterioração da capacidade do eritrócito em carrear O (HESS \& GREENWALT, 2002; KURUP et al., 2003). ${ }^{2}$

O potássio é um íon intracelular e é um importante marcador da integridade da membrana plasmática. Os seus níveis intracelulares são mantidos pela "bomba de sódio e potássio". Entretanto, baixos níveis do pH influenciam diretamente neste equilíbrio, promovendo uma migração do potássio para o meio extracelular (MEYER et al., 1995; BUSH, 2004).

O adenosina trifosfato (ATP) é um nucleotídeo presente na célula. Este é necessário no controle do balanço eletrolítico pela bomba de sódio e potássio, na manutenção da forma celular, e no aumento da flexibilidade, possuindo uma particular ação na produção de energia (SCOTT et al., 2005). Com a depleção nos seus níveis, ocorrerão alterações como perda do formato normal e aumento da fragilidade da membrana do eritrócito, ocasionando maior grau de hemólise, tanto durante a conservação como após a sua administração no paciente (EISENBRANT \&
SMITH, 1973; HÖGMAN et al., 2002; KURUP et al., 2003).

Tendo em vista as dificuldades em manter os estoques de um banco de sangue veterinário, é imperativo o conhecimento de novas técnicas de preservação do sangue canino, com fins de prolongar o tempo de conservação deste sem comprometer a manutenção de seus parâmetros bioquímicos. Além disso, em medicina veterinária, poucos estudos têm sido direcionados à conservação do sangue canino para transfusão, sendo este um dos fatores limitantes no uso terapêutico do mesmo (RIBEIRO FILHO et al., 1994).

O presente trabalho teve como objetivo avaliar e comparar as alterações bioquímicas e hemogasométricas do sangue total canino armazenado em bolsas CPDA-1 e CPD/SAG-M durante 41 dias de armazenamento

\section{MATERIAL E MÉTODOS}

Foram utilizados 14 cães adultos e em boas condições de saúde, atestados pelo exame clínico, hemograma completo e pesquisa de parasitos intestinais, sendo que todos estavam dentro das exigências para doadores de sangue.

Foram coletados aproximadamente $420 \mathrm{~mL}$ de sangue de cada animal e armazenados em bolsas CPDA-1 ${ }^{\text {a }}$ e CPD/SAG-M ${ }^{\text {a }}$. Estas foram organizadas em dois grupos com sete unidades cada. A distribuição se deu da seguinte maneira: Grupo 1 (G1), contendo sangue coletado e armazenado em bolsas CPDA-1; e Grupo 2 (G2), contendo sangue coletado e armazenado em bolsas CPD/SAG-M. Todas as bolsas foram conservadas em refrigeração de 4 a $6^{\circ} \mathrm{C}$ por um período de 41 dias.

Uma alíquota de sangue total foi retirada das bolsas de ambos os grupos no primeiro dia (D0) e nos dias sete (D7), 14 (D14), 21 (D21), 31 (D31) e 41 (D41) e submetida às análises laboratoriais. O procedimento de retirada foi todo realizado em capela microbiológica obedecendo a todas as técnicas de assepsia.

Os testes realizados foram: dosagem do DPG eritrocitário por fotometria ultravioleta ${ }^{\mathrm{b}}$; glicose plasmática por espectrofotometria colorimétrica ${ }^{\mathrm{c}}$, sódio e potássio plasmáticos por fotometria de chama ${ }^{\text {d; }}$ proteína plasmática total por medidas de refratometria mensuração da $\mathrm{pO}_{2}, \mathrm{pCO}_{2}$, bicarbonato e $\mathrm{pH}$ sanguíneos por aparelho de hemogasometria ${ }^{\mathrm{f}}$.

A avaliação estatística foi realizada ao término da coleta dos dados experimentais e analisada através do programa estatístico GraphPad InStat ${ }^{\mathrm{g}}$, 
utilizando-se para os dados paramétricos a análise de variância (ANOVA). Posteriormente, os dados que passaram pelas premissas da análise de variância foram submetidos ao teste de Tukey, com grau de significância de 5\% $(\mathrm{P}<0,05)$, que avaliou o comportamento das variáveis no decorrer do tempo.

Para a comparação entre grupos, foi realizado o teste t de Student com grau de significância de 5\% $(\mathrm{P}<0,05)$.

\section{RESULTADOS E DISCUSSÃO}

Os níveis da proteína plasmática se mantiveram estatisticamente estáveis em ambos os grupos. Entretanto, ao submeter à análise do coeficiente de variância e comparar os dois grupos, pôde-se observar uma diferença entre os grupos no $41^{\circ}$ dia de armazenagem do sangue, sugerindo que houve uma melhor preservação dos eritrócitos no G2, uma vez que o aumento da variável proteína plasmática indicaria uma maior perda de componentes intracelulares (Figura 1A).

Para a glicose plasmática, a comparação entre o D0 e o D41 em cada grupo, o G2 demonstrou menor redução desta variável ao final do período. Esta superioridade das bolsas do G2 pode ser atribuída à melhor conservação do ATP, pois, segundo AUTHENMENT et al. (1986) e HÖGMAN et al. (2002), com a queda do $\mathrm{pH}$ e um maior consumo da glicose celular, este nucleotídeo é consumido na tentativa de manter as reservas de energia da célula. Portanto, melhores níveis de ATP representam um menor consumo da glicose celular (Figura 1B). A redução das concentrações da glicose apresentada pelo G1 também foi observada por WARDROP et al. (1994), em estudos de conservação do sangue canino em bolsas CPDA-1.

O sódio plasmático, embora apresentasse um comportamento peculiar, estatisticamente não demonstrou diferenças na comparação entre grupos (Figura 1C). O G1 teve um comportamento semelhante ao G2 sem diferenças significativas. Como o sódio é um eletrólito tipicamente extracelular, pode-se sugerir que não houve perda de água para o exterior das bolsas de ambos os grupos. Estes resultados corroboram os achados de HESS \& GREENWALT (2002), quando afirmaram que o plástico das bolsas permite a saída apenas do $\mathrm{CO}_{2}$.

$\mathrm{Na}$ análise do potássio plasmático, foi encontrado um aumento significativo em ambos os grupos durante todo o período estudado. $\mathrm{Na}$ comparação entre os grupos, o G2 apresentou menores níveis deste eletrólito em relação ao G1. Este achado sugere um melhor desempenho do G2, demonstrando uma maior preservação da membrana plasmática, podendo, inclusive, ser um indicador indireto dos níveis de ATP, pois este nucleotídeo está intimamente relacionado à manutenção da membrana plasmática, sendo, também, responsável pelo equilíbrio eletrolítico da célula (Figura 1D).

Notou-se que os níveis do potássio plasmático não excederam a 5,1 e 3,8mEqL ${ }^{-1}$ para G1 e G2 respectivamente. Estes achados contrariam os de KURUP et al. (2003), quando, em estudo com sangue humano conservado em bolsas CPD/SAG-M por 28 dias de refrigeração, encontraram médias de $61 \mathrm{mEqL}^{-1}$ no último dia de armazenagem. Entretanto, estes resultados achados confirmam as observações de KERR (2003) e de KORTE \& VERHOEVEN (2004), quando afirmaram que, em cães, os níveis intracelulares do potássio são menores, quando comparado a outras espécies, inclusive o homem.

Também foi observada uma forte correlação entre as variáveis potássio plasmático e pH (Figura $1 \mathrm{~F}$ ), em que se constatou um alto coeficiente de correlação para o G1 e o G2 em função do pH (r=-0,92449 e r=-0,9375 respectivamente). Tal estudo demonstra que o aumento do potássio plasmático nos grupos G1 e G2 foi em $85 \%$ e $88 \%$ respectivamente, explicados pela queda do $\mathrm{pH}$ no interior das bolsas. Estes dados corroboram a observação de BUSH (2004), quando afirmou que a membrana plasmática é diretamente afetada pela redução do $\mathrm{pH}$, influenciando, desta forma, na migração do potássio contido no fluído intracelular para o meio extracelular.

O DPG apresentou um declínio semelhante em ambos os grupos. Porém, nos primeiros sete dias, foi observado um aumento significativo, igual para o G1 e o G2, estabilizado no sétimo dia de armazenagem. Estes dados contrariam os achados de EISENBRANDT \& SMITH (1973), SMITH et al. (1978), em estudo do comportamento bioquímico do sangue canino armazenado em solução de ácido cítrico e dextrose e das observações feitas por D'ALMEIDA et al. (2000), em sangue humano e de ratos conservados em bolsas CPDA-1, quando afirmaram que o DPG sofre um declínio já nos primeiros dias. Segundo WARDROP et al. (1994), HESS \& GREENWALT (2002) e KURUP et al. (2003), ocorre um colapso na produção do DPG em valores de $\mathrm{pH}$ inferiores a sete. Entretanto, neste estudo, foi observado um aumento do DPG entre os dias zero e sete, com níveis do $\mathrm{pH}$ abaixo de sete em ambos os grupos. Após este período, houve uma queda contínua dos valores do DPG (Figura 1E).

A explicação para o aumento nos níveis do DPG após o dia zero, alcançando picos no 7ํ dia, mesmo 

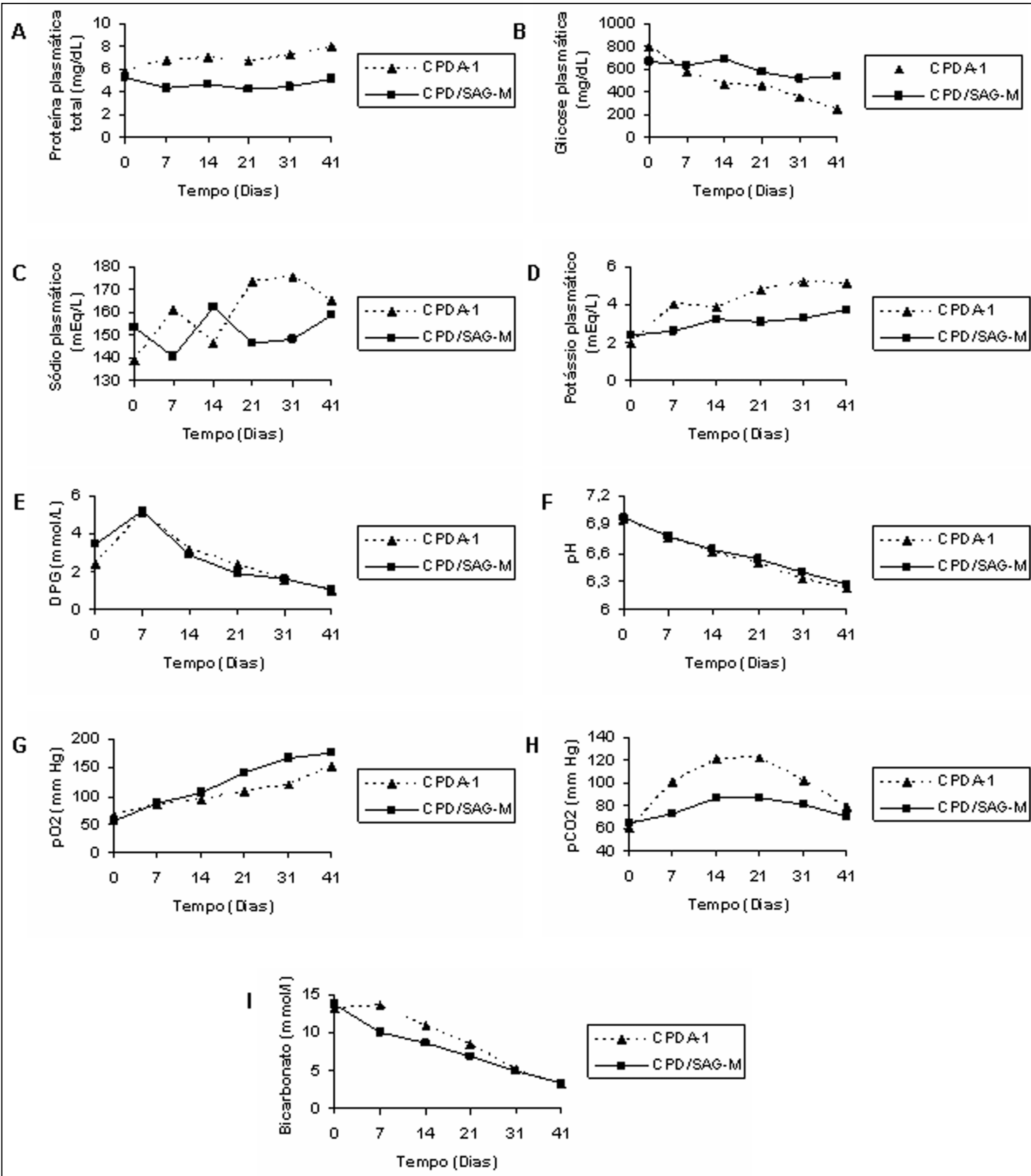

Figura 1 - Médias semanais para: (A) proteína total; (B) glicose; (C) sódio; (D) potássio; (E) DPG; (F) pH; (G) pO ; (H) pCO ; (I) bicarbonato para sangue canino armazenado em bolsas CPDA-1 e CPD/SAG-M.

com $\mathrm{pH}$ abaixo de sete, em ambos os grupos, pode estar no fato de que as soluções conservadoras das bolsas já possuem altos níveis de adenina, energia (na forma de dextrose) e fosfato. Segundo SCOTT et al. (2005), o fosfato é substrato para a produção de mais DPG

Feita a análise de correlação para a variável DPG em função do $\mathrm{pH}$ a partir do $7^{\circ}$ dia, observou-se um coeficiente de determinação de r=0,97 para G1 e r=0,94 para G2, demonstrando que a redução do DPG está relacionada em 95\% e $88 \%$ para o G1 e o G2, respectivamente, ao declínio do $\mathrm{pH}$ no interior das bolsas.

Em ambos os grupos, foram observados um decréscimo do $\mathrm{pH}$, entretanto, sem diferenças entres estes. Os valores do pH ficaram em torno de 6,96 e 6,97 para o G1 e G2, respectivamente, corroborando os achados de WARDROP et al. (1994), em estudo do 
sangue canino armazenado em bolsas CPDA-1. Porém, contrariando os achados de KURUP et al. (2003) em estudo com sangue humano conservado em bolsas CPDA-1 e CPD/SAG-M, o qual obteve médias do $\mathrm{pH}$ de 7,38 e 7,36, respectivamente.

$\mathrm{O}$ decréscimo do $\mathrm{pH}$, no primeiro dia, confirma os achados de RIBEIRO FILHO et al. (1994) e HESS et al. (2002), que observaram uma redução do pH ainda no primeiro dia de armazenamento. Possivelmente isso se deve à acidez da solução contida nas bolsas. $\mathrm{O}$ decréscimo do pH no decorrer do tempo ocorreu, provavelmente, pelos metabólicos ácidos provenientes do metabolismo anaeróbico dos eritrócitos durante a conservação (Figura 1F).

Houve aumento da $\mathrm{pO}_{2}$ em ambos os grupos. Este dado corrobora os resultados encontrados por RIBEIRO FILHO et al. (1994), os quais, em estudos com sangue bovino em bolsas CPDA-1, relataram que o plástico das bolsas é permeável ao $\mathrm{O}_{2}$. Este fato pode explicar o aumento dos níveis da $\mathrm{pO}_{2}$ no sangue. Entretanto, contraria as afirmações de HÖGMAN et al. (2002), quando relataram que o plástico é permeável apenas ao $\mathrm{CO}_{2}$, sendo pobremente transpassado por outros gases.

Embora não tenham sido observadas diferenças significativas entre os valores do $41^{\circ}$ dia entre os grupos, é possível observar que o G2 apresentou uma discreta melhora nos níveis da $\mathrm{pO}_{2}$, sugerindo que o sangue, deste grupo, disponibilize uma concentração maior de $\mathrm{O}_{2}$ para o paciente transfundido (Figura 1-G).

No estudo da $\mathrm{pCO}_{2}$, foi observado um aumento significativo dos níveis apenas no G1 até os dias 14 e 21, reduzindo após este período. Esta variação do G1 também foi observada por RIBEIRO FILHO et al. (1994), que estudaram o sangue bovino em bolsas CPDA-1. Entretanto, foi observado que os valores da $\mathrm{pCO}_{2}$ no $\mathrm{G} 1$ foram superiores aos valores observados no G2 nos dias sete, 14 e 21. Segundo HÖGMAN et al. (2002), altos níveis da pCO $\mathrm{pC}_{2}$ expõem o DPG a uma maior taxa de degradação, provavelmente por competir por seu sítio de ligação na hemoglobina. Mesmo tendo observado níveis da $\mathrm{pCO}_{2}$ estatisticamente iguais em ambos os grupos no $41^{\circ}$ dia, no G1, a pressão parcial do $\mathrm{CO}_{2}$ se elevou significativamente mais que no G2 nos dias sete, 14 e 21, expondo, neste período, a uma maior degradação do DPG. Com base nestes achados, é possível afirmar que, por ter estatisticamente mantido estáveis os níveis da $\mathrm{pCO}_{2}$ no decorrer do período, o G2 apresentou um melhor desempenho (Figura 1H).

No estudo do bicarbonato plasmático $\left(\mathrm{HCO}_{3}^{-}\right)$, foi notado um declínio gradual e semelhante para ambos os grupos estudados no decorrer do tempo
(Figura 1I). Segundo LUNA (2002), o bicarbonato é uma base fraca, responsável por mais de $50 \%$ da capacidade tamponante do meio extracelular. Por isso, esta redução nos valores sangüíneos do $\mathrm{HCO}_{3}{ }^{-}$pode ser explicada por um consumo deste íon no controle da acidez, reduzindo os seus níveis na mesma proporção em que há a produção de ácidos metabólicos no interior das bolsas. Este declínio do bicarbonato também foi encontrado por RIBEIRO FILHO et al. (1994), em estudo com sangue bovino conservado em bolsas CPDA-1.

\section{CONCLUSÕES}

Com base nos estudos realizados, é possível afirmar que o DPG continua a ser produzido mesmo em $\mathrm{pH}$ inferior a sete, por, pelo menos, sete dias de armazenamento. Além disso, as concentrações do potássio plasmático não se elevam na mesma proporção das encontradas em sangue humano conservado em bolsas CPD/SAG-M e também ocorre aumento da $\mathrm{pCO}_{2}$ no sangue conservado nas bolsas CPDA-1. Portanto, é mais indicado o uso das bolsas CPD/SAG-M para a conservação do sangue canino sob refrigeração em um período máximo de 41 dias de armazenagem em relação às bolsas CPDA-1.

\section{FONTES DE AQUISIÇÃO}

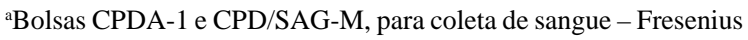
hemocare.

${ }^{\mathrm{b}}$ Roche Diagnostics - Germany/30 determinações - UV.

'Glicose Enzimática GOD-RAP Cristal - 500 testes K. 054 BIOCLIN.

${ }^{\mathrm{d}}$ Fotômetro de Chama Micronel - B 462.

eRefratômetro: Atago, Hand Refractometer, Mod. Uricon cat n. 135.

${ }^{\mathrm{f}}$ Gasômetro ABL 5 - Radiometer/Copen Hagen.

gGraphPad InStat 3.00 para Windows 98 - GraphPad Software, www.graphpad.com.

\section{AGRADECIMENTOS}

À Fundação de Amparo à Pesquisa do Estado de Minas Gerais (FAPEMIG), pelo financiamento deste estudo.

\section{COMITÊ DE ÉTICA E BIOSSEGURANÇA}

Este estudo foi submetido à apreciação do comitê de ética e biossegurança do Departamento de Veterinária da Universidade Federal de Viçosa - UFV, sendo aprovado e protocolado como processo 28/2005 no dia 07 de junho de 2005.

\section{REFERÊNCIAS}

AUTHEMENT, J.M. et al. Canine blood component therapy: product preparation, storage and administration. Journal of American Animal Hospital Association, v.23, p.483-493, 1986.

Ciência Rural, v.38, n.2, mar-abr, 2008. 
BROOKS, M. Transfusion medicine. In: MURTAUGH, R.J.; KAPLAN, P.M. Veterinary emergency and critical care medicine. Saint Louis: Mosby Yearbook, 1992. 935p.

BUNN, H.F. Differences in interactions of 2,3diphosphoglicerate with certain mammalian hemoglobin. Science, v.23, p.1049-1050, 1971.

BUSH, B.M. Eletrólitos e metais. In: Interpretação de resultados laboratoriais para clínicos de pequenos animais. São Paulo: Roca, 2004. cap.7, p.262-289.

CHIARAMONTE, D. Blood-component therapy: selection, administration and monitoring. Clinical Techniques in Small Animal Practice, v.19, n.2, p.63-67, 2004.

D’ALMEIDA, M.S. et al. A comparison of biochemical and functional alterations of rat and human erythrocytes stored in CPDA-1 for 29 days: implications for animal models of transfusions. Transfusión Medicine, v.10, p.291-303, 2000.

EISENBRANDT, D.L.; SMITH, J.E. Use of biochemical measures to estimate viability of red blood cells in canine blood storage in acid citrate dextrose solution, with and without added ascorbic acid. Journal of American Animal Hospital Association, v.163, n.8, p.984-987, 1973.

HESS, J.R.; GREENWALT, T.J. Storage of blood cells: new approaches. Transfusion Medicine Reviews, v.16, n.4 p.283-295, 2002.

HESS, J.R. et al. Alkaline CPD and the preservation of RBC 2,3-DPG. Transfusion, v.42, p.747-752, 2002.

HÖGMAN, C.F. et al. Improved maintenance of 2,3 DPG and ATP in RBC's stored in a modified additive solution. Transfusion, v.42, p.824-829, 2002.

KERR, M.G. Eletrólitos. In: Exames laboratoriais em medicina veterinária: bioquímica clínica e hematologia. 2.ed. São Paulo: Roca. 2003. cap.5, p.95106.

KORTE, D.; VERHOEVEN, A.J. Quality determinants of erythrocyte destined for transfusion. Cellular and Molecular Biology, v.50, n.2, p.187-195, 2004.

KRISTENSEN, A.T.; FELDMAN, B.F. Bancos de sangue e medicina transfusional. In: ETTINGER, E.J.; FELDMAN, E.C. (Ed.). Tratado de medicina interna veterinária. 4.ed. Barueri: Manole, 1997. v.1, p.497-517.
KURUP, P.A. et al. Modified formulation of CPDA for storage of whole, and of SAGM for storage of red blood cells, to maintain the concentration of 2,3-diphosphoglycerate. Vox Sanguinis, v.85, p.253-261, 2003.

LOPES, R.S. et al. Alterações hematológicas e bioquímicas em sangue total de eqüinos, conservado para transfusão em bolsas plásticas com ACD-F. Revista Universidade Rural: Ciência da vida, v.17, p.91-94, 1995.

LUCAS, R.L. et al. Collection and preparation of blood products. Clinical Techniques in Small Animal Practice, v.19, n.2, p.55-62, 2004.

LUNA, S.P.L. Equilíbrio ácido-básico. In: FANTONI, D.T.; CORTOPASSI, S.R.G. (Ed.). Anestesia em cães e gatos. São Paulo: Roca, 2002. cap.10, p.120-129.

MEYER, D.J. et al. Homeostasia e distúrbios eletrolíticos e ácido-básicos. In: ____ Medicina de laboratório veterinário: interpretação e diagnóstico. São Paulo: Roca, 1995. p.83-90.

NUSBACHER, J. Blood transfusion is mononuclear cell transplantation. Transfusion, v.34, p.1002-1004, 1994.

RIBEIRO FILHO, J.D. et al. Alterações hemogasométrias de sangue bovino durante a conservação em frascos de vidro com ACD e bolsas plásticas com CPDA-1, por 35 dias. Veterinary Zootecnic, v.6, p.77-84, 1994

ROZANSKI, E; LAFORCADE, A.M. Transfusion medicine in veterinary emergency and critical care medicine. Clinical Techniques in Small Animal Practice, v.19, n.2, p.83-87, 2004

SCOTT, K.L. et al. Biopreservation of blood cells: past, present, and future. Transfusion Medicine Reviews, v.19, n.2, p.127142, 2005

SMITH, J.E. et al. A new storage medium for canine blood. American Veterinary Medical Associacion, v.15, p.701703, 1978

WARDROP, K.J. et al. An in vitro evaluation of storage media for the preservation of canine packed red blood cells. Veterinary Clinical Pathology, v.23, p.83-88, 1994.

WILLER, R.L.; RIEDESEL, D.H. Transfusion therapy and blood banking in the dog and cat. Iowa State University Veterinary, v.47, p.102-109, 1985. 